TITLE:

\title{
Visualization of magnetic microparticles in liquid and control of their motion using dynamic magnetic field
}

\section{$\operatorname{AUTHOR}(\mathrm{S}):$}

Tokura, S.; Hara, M.; Kawaguchi, N.; Izawa, J.; Amemiya, $\mathrm{N}$.

\section{CITATION:}

Tokura, S. ... [et al]. Visualization of magnetic microparticles in liquid and control of their motion using dynamic magnetic field. JOURNAL OF APPLIED PHYSICS 2010, 107(9): 09B521.

\section{ISSUE DATE:}

2010-05

URL:

http://hdl.handle.net/2433/147199

\section{RIGHT:}

Copyright 2010 American Institute of Physics. This article may be downloaded for personal use only. Any other use requires prior permission of the author and the American Institute of Physics. The following article appeared in JOURNAL OF APPLIED PHYSICS107, 09B521 (2010) and may be found at 


\title{
Visualization of magnetic microparticles in liquid and control of their motion using dynamic magnetic field
}

\author{
S. Tokura, ${ }^{1,2, a)}$ M. Hara, ${ }^{1}$ N. Kawaguchi, ${ }^{1}$ J. Izawa, ${ }^{1}$ and N. Amemiya ${ }^{2}$ \\ ${ }^{1}$ Research Laboratory, IHI Corporation, 1 Shin-Nakahara, Isogo, Yokohama, Kanagawa 235-8501, Japan \\ ${ }^{2}$ Department of Electrical Engineering, Kyoto University, Kyoto-Daigaku-Katsura, Nishikyo, Kyoto \\ 615-8510, Japan
}

(Presented 22 January 2010; received 31 October 2009; accepted 18 January 2010; published online 6 May 2010)

\begin{abstract}
Visualization technique of micro- or nanoparticles with sufficient spatial and temporal resolutions is required for quantitative study of motion of magnetic particles and their control using a dynamic magnetic field. Based on the particle tracking velocimetry method, a visualization system of micron-size particles has been developed. A proof-of-concept experiment of controlling magnetic particles using a dynamic magnetic field was made, and motions of magnetic particles under a dynamic magnetic field were visualized successfully using the developed system: the motions of particles synchronizing with the applied dynamic magnetic field could be observed. () 2010 American Institute of Physics. [doi:10.1063/1.3358614]
\end{abstract}

\section{INTRODUCTION}

Motions of magnetic micro- or nanoparticles have been attracted interests, in some areas of biotechnology such as cell separation or drug delivery. ${ }^{1,2}$ Many authors reported chainlike cluster formation or magnetic accumulation of the particles from macroscopic viewpoints such as morphology or accumulation ratio. ${ }^{3-7}$

Furlani $^{8}$ reported analytical analysis of particle transport in a magnetophoretic microsystem and estimated the capture efficiency considering fluidic forces, magnetic forces, and Brownian motion. Warnke ${ }^{9}$ also studied the separation of magnetic particles in fluid with a finite-element analysis considering these forces. As for experimental studies, Kikura et $a l .{ }^{10,11}$ reported the flow visualization of primary agglomerated magnetic particles considering Brownian motion. Erglis et al. ${ }^{12}$ reported dynamics of magnetotactic bacteria in a rotating magnetic field. However, quantitative study of motion of magnetic particles and their control using a dynamic magnetic field have not been enough yet. Visualization technique of micro- or nanoparticles with sufficient spatial and temporal resolutions is required for considering the electromagnetic interaction between particles or considering various forces and fields which can influence motions of magnetic particles.

In this study, we developed a visualization system of microparticles and used it for a proof-of-concept experiment of controlling magnetic particles using a dynamic magnetic field.

\section{EXPERIMENTS}

\section{A. System for visualization of microparticles}

The particle tracking velocimetry (PTV) method with green laser [second-harmonic generation of Nd:YAG (yttrium aluminum garnet) laser, $20 \mathrm{~mW}, 532 \mathrm{~nm}, \mathrm{CW}$ ] illumi-

\footnotetext{
${ }^{a)}$ Author to whom correspondence should be addressed. Electronic mail: susumu_tokura@ihi.co.jp.
}

nation or the halogen lamp illumination was applied to visualization of motions of magnetic microparticles (ferrite particle, $\phi 12 \mu \mathrm{m})$ in liquid in a small vessel. Antiferromagnetic particles $\left(\alpha-\mathrm{Fe}_{2} \mathrm{O}_{3}, \phi 5 \mu \mathrm{m}\right)$ were used as a reference particle which indicated almost fluid flow in the vessel. The apparatuses using the halogen lamp illumination system and the laser illumination system are shown in Fig. 1. The halogen lamp illuminated particles from the back of the vessel or the laser illuminated particles from the side of the vessel, while motions of microparticles were recorded by an image analyzer from the front of the vessel. The recorded data were analyzed to determine the displacement and velocity of each particle.

\section{B. Control of magnetic particles using a dynamic magnetic field and their visualization}

As shown in Fig. 1, using the LABVIEW software, a signal was generated and the image analyzer was triggered to record. The signal generated by the software drew power from the power supply and were used to power the electromagnetic coil above and below the microparticle-containing

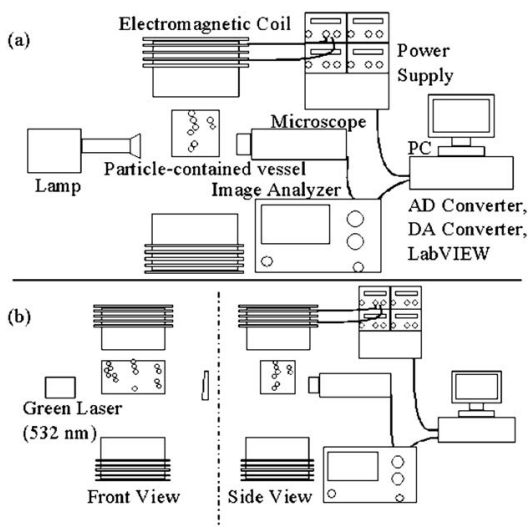

FIG. 1. Visualization system of the particle motion using (a) the lamp illumination (halogen lamp, $100 \mathrm{~W}$ ) and (b) the green laser (wavelength of the laser: $532 \mathrm{~nm}$ ). 
TABLE I. Experimental conditions for the control of motion of magnetic particles.

\begin{tabular}{lc}
\hline \hline Solvent & $\begin{array}{c}\text { Water } \\
\text { Quartz vessel: diameter } \phi 50 \mathrm{~mm} / \\
\text { length } 50 \mathrm{~mm}\end{array}$ \\
Yessel & $\begin{array}{c}\text { Yoke: window flame shape, coil: } \\
20 \mathrm{~A}(\max ) \times 500 \mathrm{turn} / \text { pole, } \\
\text { and gap: } 300 \mathrm{~mm}\end{array}$ \\
$\begin{array}{l}\text { Electromagnet } \\
\text { Surface of the magnetic pole } \\
\text { Waveform of the applied } \\
\text { magnetic flux density }\end{array}$ & $\begin{array}{r}\text { Rectangular: } 100 \times 100 \mathrm{~mm}^{2} \\
\text { Frequency of the applied } \\
\text { magnetic field }\end{array}$ \\
$\begin{array}{l}\text { Frame rate of the camera } \\
\text { and } 33-21 \mathrm{mT})\end{array}$ \\
\hline
\end{tabular}

vessel. The observation system was focused, and the video of microparticles in the vessel was recorded at a fixed height for many different voltages and waveforms. For each trial, at least one particle was tracked, and the applied voltage and the observation video were recorded synchronously. Representative experimental conditions for the control of motion of magnetic particles are summarized in Table I.

\section{RESULTS AND DISCUSSION}

\section{A. Visualization test of microparticles}

As the demonstration of visualization systems, motions of ferrite microparticles were visualized using each method (halogen lamp illumination and laser illumination). In the case of particle which diameter was larger than the wavelength of a light source, the halogen lamp (broad wavelength range) illumination was convenient, because the shapes and rugged surfaces of particles could be observed well by the diffraction scattering. In the other case, the Rayleigh light scattering is the effective technique for the tracking of nanoparticles, ${ }^{13}$ especially the laser illumination method with the Rayleigh light scattering was convenient for the easy condensation and the monochromaticity.

Images of microparticles in the liquid after shuffling the vessel are shown in Fig. 2. Figure 2(a) shows the visualization image by the halogen lamp illumination and Fig. 2(b) shows the image which was taken after $2 \mathrm{~s}$ using the laser illumination without stopping the image acquisition. As the halogen lamp illumination method was able to measure cross section of a particle accurately, this method was useful for the quantitative study of understanding the motion of magnetic microparticles under dynamic magnetic fields. On the

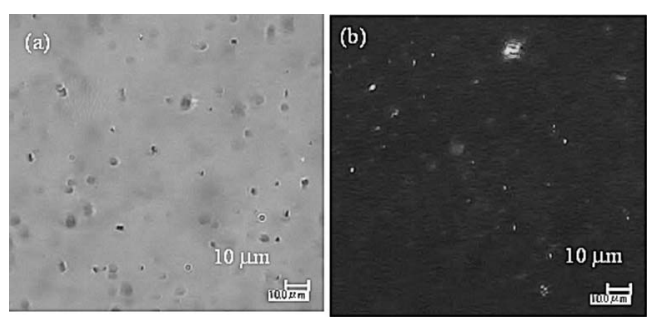

FIG. 2. Visualization images by (a) the halogen lamp illumination and by (b) the laser illumination which was taken after $2 \mathrm{~s}$ without stopping the image acquisition.

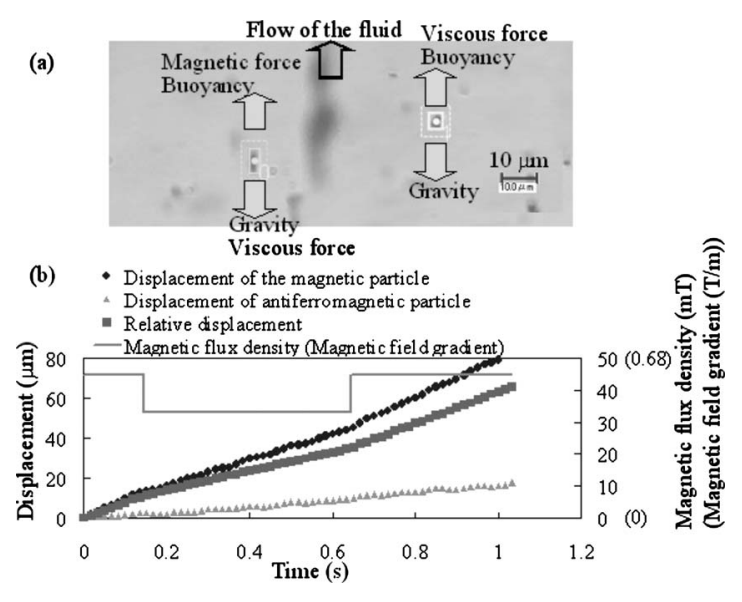

FIG. 3. (a) Visualization image of applying magnetic field (the rectangular waveform) to the magnetic particle (left) and the antiferromagnetic particle (right). (b) Relation between the applied magnetic flux density or the applied magnetic field gradient and vertical displacement of the microparticle.

other hand, the laser illumination method was useful for its good ability of tracking due to the high contrast. We will apply this method in our future study on controlling magnetic nanoparticles.

\section{B. Proof-of-concept experiments of controlling magnetic particles using a dynamic magnetic field}

We performed the basic experiment of applying dynamic magnetic field to magnetic particles with the visualization system shown in Fig. 1. Moreover, the feasibility of the quantitative study on the particle motion by the PTV method was confirmed.

Figure 3(a) shows the visualization image of applying magnetic field (the rectangular waveform) to magnetic particles (left) and antiferromagnetic particles (right, including weak magnetic particles). In this case, the flow direction of both magnetic and antiferromagnetic particles was upwards. Moreover, representative forces and fields influenced to each particle were considered to be the magnetic force, viscous force, gravity, and buoyancy. ${ }^{8,14}$ Figure 3(b) shows the relation between the applied magnetic flux density and the microparticle vertical displacement which was obtained from PTV method. The frequency was $1 \mathrm{~Hz}$, the applied magnetic flux density was 33-45 $\mathrm{mT}$, and the gradient of magnetic field at the view area was $0.45-0.6 \mathrm{~T} / \mathrm{m}$. The closed square symbol shown in Fig. 3(b) represents the relative vertical displacement between the magnetic and antiferromagnetic particle. As the motion of the magnetic particle was also influenced by fluid flow, the comparison between the applied magnetic flux density and the relative vertical displacement was to be reasonable on the case of estimating the responsibility of magnetic particles to applied magnetic force.

The relative velocities, which were calculated from the relative displacement curve shown in Fig. 3(b) of the magnetic particle under magnetic fields were about 77 (at $45 \mathrm{mT}$ ) and $48 \mu \mathrm{m} / \mathrm{s}$ (at $33 \mathrm{mT}$ ). Here, forces and fields which influenced to the magnetic particle were able to estimate using following expressions with some assumptions. ${ }^{8}$

The magnetic force is given by 


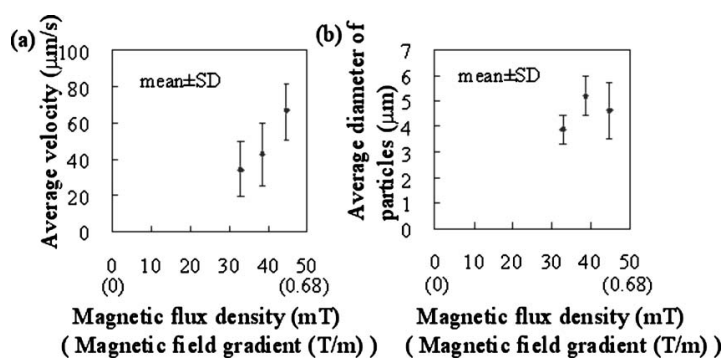

FIG. 4. (a) Relation between applied magnetic flux density or the applied magnetic field gradient and relative velocity of particles. (b) Relation between the applied magnetic flux density and the applied magnetic field gradient and average diameter of particles.

$$
F_{p}=\mu_{0} V_{p} 3 H_{a} \nabla H_{a},
$$

where $\mu_{0}$ is the permeability, $V_{p}$ is the volume of the particle, and $H_{a}$ is the applied magnetic field intensity (below saturation).

The viscous (fluidic) force is given by

$$
F_{v}=6 \pi \eta R_{p}\left(v_{p}-v_{f}\right),
$$

where $\eta$ and $v_{f}$ are the viscosity and the velocity of the fluid, respectively. $R_{p}$ and $v_{p}$ are the radius and the velocity of the particle, respectively.

The gravitational and buoyant forces are given by

$$
\begin{aligned}
& F_{g}=\rho_{p}(4 / 3) \pi R_{p}^{3} g, \\
& F_{b}=\rho_{f}(4 / 3) \pi R_{p}^{3} g,
\end{aligned}
$$

where $\rho_{p}$ and $\rho_{f}$ are the density of the particle and the fluid.

From expression (1)-(4), we obtained $F_{p}=6.6 \mathrm{pN}, F_{v}$ $=4.2 \mathrm{pN}, F_{g}=2.0 \mathrm{pN}$, and $F_{b}=1.0 \mathrm{pN}\left(F_{p}+F_{b}>F_{v}+F_{g}\right.$ @ $45 \mathrm{mT}, 0.6 \mathrm{~T} / \mathrm{m})$. We also obtained $F_{p}=3.1 \mathrm{pN}, F_{v}$ $=2.6 \mathrm{pN}, F_{g}=2.0 \mathrm{pN}$, and $F_{b}=1.0 \mathrm{pN}\left(F_{p}+F_{b}<F_{v}+F_{g}\right.$ @ $33 \mathrm{mT}, 0.45 \mathrm{~T} / \mathrm{m})$. These result indicated that the magnetic particle was able to be dragged by the magnetic force at higher magnetic field gradient $(0.6 \mathrm{~T} / \mathrm{m})$, as shown in Fig. 3(b). However, further examination will need to make clear the influence of the particle shape or fluid flow occurred by the other particles.

Figure 4 shows the relation between applied magnetic flux density (magnetic field gradient) and relative velocity of particles which were calculated from the vertical displacement curve shown in [Fig. 3(b), closed square symbol line]. Where, the waveform of magnetic flux density was rectangular wave (three cases: 45-33, 39-27, and 33-21 mT). The frequency of the applied magnetic flux density was $1 \mathrm{~Hz}$. As a result, the relative velocity of magnetic particles had the tendency of being increased in proportion to the applied magnetic flux density and the magnetic field gradient. However, the error bar of the relative velocity was large mainly due to the dispersion of the average diameter of particles, as shown in Fig. 4(b).

The responsibility of magnetic particles to the applied magnetic force was confirmed. Figure 5 shows the typical relation curve between the relative vertical displacement of the magnetic particle and the applied magnetic flux density [(a) and (b): lamp illumination, $2 \mathrm{~Hz}, 33-45 \mathrm{mT}$, and 0.45$0.6 \mathrm{~T} / \mathrm{m}$, (c) and (d): laser illumination, $1 \mathrm{~Hz}, 33-45 \mathrm{mT}$, and

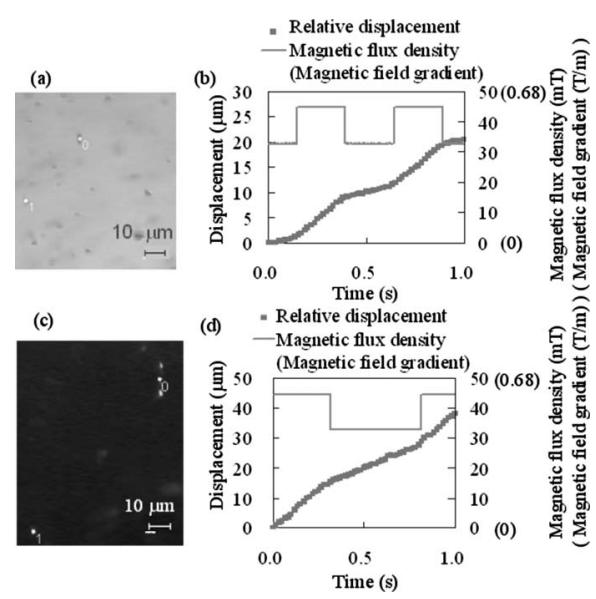

FIG. 5. Relation between applied magnetic flux density or the applied magnetic field gradient and relative vertical displacement of the particle, using [(a) and (b)] lamp illumination ( $2 \mathrm{~Hz}, 33-45 \mathrm{mT}$, and 0.45-0.6 T/m), and [(c) and (d)] laser illumination $(1 \mathrm{~Hz}, 33-45 \mathrm{mT}$, and $0.45-0.6 \mathrm{~T} / \mathrm{m})$.

0.45-0.6 T/m]. As shown in Figs. 5(b) and 5(d), gradients of the relative vertical displacement curves were changed responding to switching the applied magnetic flux density or the applied magnetic field gradient. Therefore, the motion of the particle was well synchronized to the frequency of the rectangular wave.

\section{SUMMARY}

Based on the PTV method, we developed a visualization system of particle, which enables us to visualize the micronsize particles. A proof-of-concept experiment of controlling magnetic particles using a dynamic magnetic field was made, and motions of magnetic particles under a dynamic magnetic field were visualized successfully using the developed system: the motions of particles synchronizing with the applied dynamic magnetic field could be observed.

\section{ACKNOWLEDGMENTS}

The authors thank Mr. Troy Tamas of Massachusetts Institute of Technology for the experimental assistance.

${ }^{1}$ X. L. Li, K. L. Yao, H. R. Liu, and Z. L. Liu, J. Magn. Magn. Mater. 311, 481 (2007)

${ }^{2}$ H. Xu, T. Song, X. Bao, and L. Hu, J. Magn. Magn. Mater. 293, 514 (2005).

${ }^{3}$ F. Tsumori, M. Hirata, and S. Shima, J. Jpn. Soc. Powder Powder Metall. 52, 3 (2004).

${ }^{4}$ T. Ohara, IEEE Trans. Magn. 20, 436 (1984).

${ }^{5}$ F. Mishima, S. Fujimoto, S. Takeda, Y. Izumi, and S. Nishijima, J. Magn. Magn. Mater. 310, 2883 (2007).

${ }^{6}$ J. Cai, Y. Li, X. Li, and D. Zhang, J. Magn. Magn. Mater. 246, 36 (2002). ${ }^{7}$ S. V. Gorobets and I. A. Melnichuk, J. Magn. Magn. Mater. 182, 61 (1998).

${ }^{8}$ E. P. Furlani, J. Appl. Phys. 99, 024912 (2006).

${ }^{9}$ K. C. Warnke, IEEE Trans. Magn. 39, 1771 (2003).

${ }^{10}$ H. Kikura, J. Matsushita, O. Hirashima, M. Aritomi, and I. Nakatani, J. Magn. Magn. Mater. 289, 392 (2005).

${ }^{11}$ H. Kikura, J. Matsushita, N. Kakuta, M. Aritomi, and Y. Kobayashi, J. Mater. Process. Technol. 181, 93 (2007).

${ }^{12}$ K. Erglis, Q. Wen, V. Ose, A. Zeltins, A. Sharipo, P. Janmey, and A. Cebers, Biophys. J. 93, 1402 (2007).

${ }^{13}$ T. T. Le, P. Saveyn, H. D. Hoa, and P. V. Meeren, Int. Dairy J. 18, 1090 (2008).

${ }^{14}$ C. Tsouris and S. Yiacoumi, Sep. Sci. Technol. 32, 599 (1997). 